Louisiana State University

LSU Digital Commons

$12-1-2020$

\title{
Improving the robustness of the advanced LIGO detectors to earthquakes
}

\author{
E. Schwartz \\ LIGO Livingston
}

A. Pele

LIGO Livingston

J. Warner

LIGO Hanford

B. Lantz

Stanford University

J. Betzwieser

LIGO Livingston

See next page for additional authors

Follow this and additional works at: https://digitalcommons.Isu.edu/physics_astronomy_pubs

\section{Recommended Citation}

Schwartz, E., Pele, A., Warner, J., Lantz, B., Betzwieser, J., Dooley, K., Biscans, S., Coughlin, M., Mukund, N., Abbott, R., Adams, C., Adhikari, R., Ananyeva, A., Appert, S., Arai, K., Areeda, J., Asali, Y., Aston, S., Austin, C., Baer, A., Ball, M., Ballmer, S., Banagiri, S., Barker, D., Barsotti, L., Bartlett, J., Berger, B., Bhattacharjee, D., Billingsley, G., Blair, C., Blair, R., Bode, N., \& Booker, P. (2020). Improving the robustness of the advanced LIGO detectors to earthquakes. Classical and Quantum Gravity, 37 (23) https://doi.org/10.1088/ $1361-6382 / a b b c 8 c$

This Article is brought to you for free and open access by the Department of Physics \& Astronomy at LSU Digital Commons. It has been accepted for inclusion in Faculty Publications by an authorized administrator of LSU Digital Commons. For more information, please contact ir@lsu.edu. 


\section{Authors}

E. Schwartz, A. Pele, J. Warner, B. Lantz, J. Betzwieser, K. L. Dooley, S. Biscans, M. Coughlin, N. Mukund, R. Abbott, C. Adams, R. X. Adhikari, A. Ananyeva, S. Appert, K. Arai, J. S. Areeda, Y. Asali, S. M. Aston, C. Austin, A. M. Baer, M. Ball, S. W. Ballmer, S. Banagiri, D. Barker, L. Barsotti, J. Bartlett, B. K. Berger, D. Bhattacharjee, G. Billingsley, C. D. Blair, R. M. Blair, N. Bode, and P. Booker 


\section{PAPER}

\section{Improving the robustness of the advanced LIGO detectors to earthquakes}

To cite this article: E Schwartz et al 2020 Class. Quantum Grav. 37235007

View the article online for updates and enhancements.

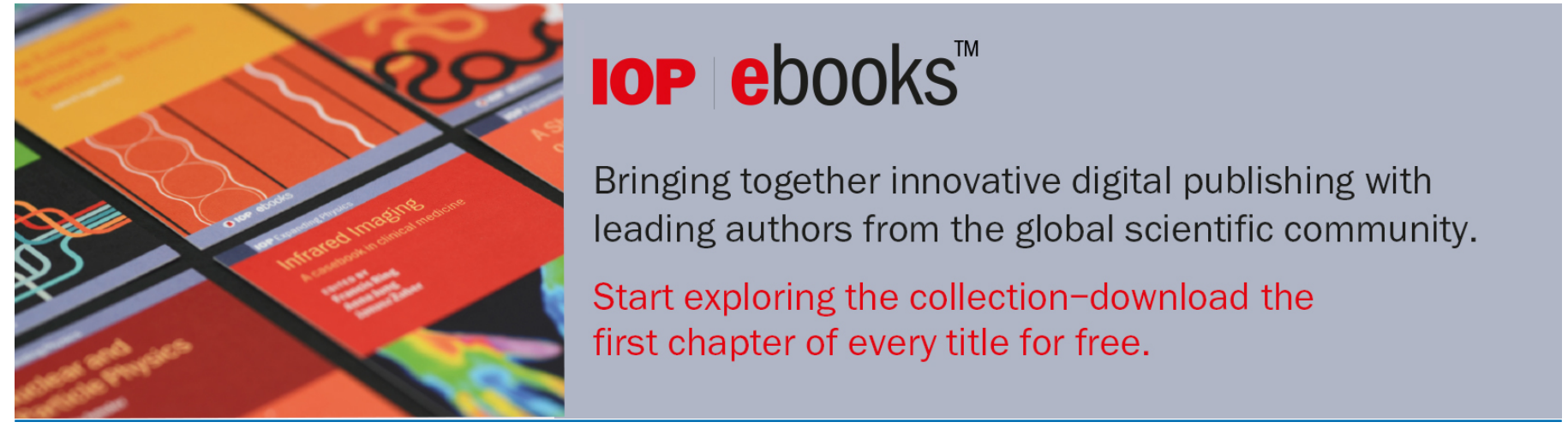

This content was downloaded from IP address 131.215 .249 .55 on 10/11/2020 at 17:34 


\title{
Improving the robustness of the advanced LIGO detectors to earthquakes
}

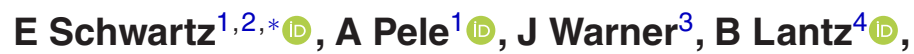

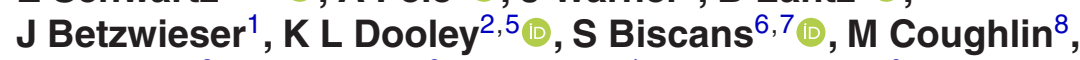 \\ N Mukund ${ }^{9}{ }^{\circ}$, R Abbott $^{6}$, C Adams $^{1}$, R X Adhikari ${ }^{6}$, \\ A Ananyeva $^{6}$, S Appert ${ }^{6}, \mathrm{~K} \mathrm{Arai}^{6}$, J S Areeda ${ }^{10}, \mathrm{Y} \mathrm{Asali}^{11}$, \\ S M Aston ${ }^{1}$, C Austin ${ }^{12}$, A M Baer ${ }^{13}$, M Ball $^{14}$, S W Ballmer ${ }^{15}$, \\ S Banagiri ${ }^{8}$, D Barker ${ }^{3}$, L Barsotti ${ }^{7}$, J Bartlett ${ }^{3}$, B K Berger ${ }^{4}$, \\ D Bhattacharjee ${ }^{16}$, G Billingsley ${ }^{6}$, C D Blair ${ }^{1}$, R M Blair ${ }^{3}$, \\ N Bode ${ }^{9,17}$, P Booker ${ }^{9,17}$, R Bork $^{6}$, A Bramley ${ }^{1}$, A F Brooks ${ }^{6}$, \\ D D Brown ${ }^{18}$, A Buikema ${ }^{7}$, C Cahillane $^{6}, \mathrm{~K} \mathrm{C} \mathrm{Cannon}^{19}$, \\ X Chen ${ }^{20}$, A A Ciobanu ${ }^{18}$, F Clara ${ }^{3}$, S J Cooper ${ }^{21}$, \\ K R Corley ${ }^{11}$, S T Countryman ${ }^{11}$, P B Covas ${ }^{22}$, D C Coyne ${ }^{6}$, \\ L E H Datrier ${ }^{23}$, D Davis ${ }^{15}$, C Di Fronzo ${ }^{21}$, J C Driggers ${ }^{3}$, \\ P Dupej $^{23}$, S E Dwyer ${ }^{3}$, A Effler ${ }^{1}$, T Etzel ${ }^{6}$, M Evans ${ }^{7}$, \\ T M Evans ${ }^{1}$, J Feicht ${ }^{6}$, A Fernandez-Galiana ${ }^{7}$, P Fritschel $^{7}$, \\ V V Frolov ${ }^{1}$, P Fulda $^{24}$, M Fyffe ${ }^{1}$, J A Giaime ${ }^{12,1}$, \\ K D Giardina1, P Godwin ${ }^{25}$, E Goetz ${ }^{12,16}$, S Gras ${ }^{7}$, C Gray ${ }^{3}$, \\ R Gray ${ }^{23}$, A C Green ${ }^{24}$, Anchal Gupta ${ }^{6}$, E K Gustafson ${ }^{6}$, \\ R Gustafson ${ }^{26}$, J Hanks ${ }^{3}$, J Hanson ${ }^{1}$, T Hardwick ${ }^{12}$, \\ R K Hasskew ${ }^{1}$, M C Heintze ${ }^{1}$, A F Helmling-Cornell ${ }^{14}$, \\ N A Holland ${ }^{27}$, J D Jones ${ }^{3}$, S Kandhasamy ${ }^{28}$, S Karki ${ }^{14}$, \\ M Kasprzack ${ }^{6}$, K Kawabe ${ }^{3}$, N Kijbunchoo ${ }^{27}$, P J King ${ }^{3}$, \\ J S Kissel ${ }^{3}$, Rahul Kumar ${ }^{3}$, M Landry ${ }^{3}$, B B Lane ${ }^{7}$, \\ M Laxen ${ }^{1}$, Y K Lecoeuche ${ }^{3}$, J Leviton ${ }^{26}$, J Liu ${ }^{9,17}$, \\ M Lormand ${ }^{1}$, A P Lundgren ${ }^{29}$, R Macas ${ }^{22}$, M Macinnis ${ }^{7}$, \\ D M Macleod ${ }^{22}$, G L Mansell ${ }^{3,7}$, S Márka ${ }^{11}$, Z Márka ${ }^{11}$, \\ D V Martynov ${ }^{21}$, K Mason ${ }^{7}$, T J Massinger ${ }^{7}$, F Matichard ${ }^{6,7}$, \\ N Mavalvala7, R McCarthy' ${ }^{3}$, E E McClelland ${ }^{27}$, \\ S McCormick ${ }^{1}$, L McCuller ${ }^{7}$, J Mclver ${ }^{6}$, T McRae $^{27}$, \\ G Mendell $^{3}$, K Merfeld $^{14}$, E L Merilh ${ }^{3}$, F Meylahn $^{9,17}$, \\ T Mistry ${ }^{30}$, R Mittleman ${ }^{7}$, G Moreno $^{3}$, C M Mow-Lowry21, \\ S Mozzon ${ }^{29}$, A Mullavey ${ }^{1}$, T J N Nelson ${ }^{1}$, P Nguyen $^{14}$, \\ L K Nuttall ${ }^{29}$, J Oberling ${ }^{3}$, Richard J Oram ${ }^{1}$, C Osthelder $^{6}$, \\ D J Ottaway ${ }^{18}$, H Overmier ${ }^{1}$, J R Palamos ${ }^{14}$, W Parker $^{1,31}$, \\ E Payne $^{32}$, C J Perez ${ }^{3}$, M Pirello $^{3}$, H Radkins ${ }^{3}$, \\ K E Ramirez ${ }^{33}$, J W Richardson ${ }^{6}$, K Riles $^{26}$,
}




\begin{abstract}
N A Robertson ${ }^{6,23}$, J G Rollins ${ }^{6}$, C L Romel ${ }^{3}$, J H Romie ${ }^{1}$, M P Ross ${ }^{34}$, K Ryan ${ }^{3}$, T Sadecki ${ }^{3}$, E J Sanchez ${ }^{6}$, L E Sanchez ${ }^{6}$, T R Saravanan ${ }^{28}$, R L Savage ${ }^{3}$, D Schaetzl ${ }^{6}$, R Schnabel ${ }^{35}$, R M S Schofield ${ }^{14}$, D Sellers ${ }^{1}$, T Shaffer ${ }^{3}$, D Sigg ${ }^{3}$, B J J Slagmolen ${ }^{27}$, J R Smith ${ }^{10}$, S Soni ${ }^{12}$, B Sorazu ${ }^{23}$, A P Spencer ${ }^{23}$, K A Strain ${ }^{23}$, L Sun 6 , M J Szczepańczyk ${ }^{24}$, M Thomas ${ }^{1}$, P Thomas $^{3}$, K A Thorne ${ }^{1}$, K Toland $^{23}$, C I Torrie ${ }^{6}$, G Traylor ${ }^{1}$, M Tse ${ }^{7}$, A L Urban ${ }^{12}$, G Vajente $^{6}$, G Valdes ${ }^{12}$, D C Vander-Hyde ${ }^{15}$, P J Veitch ${ }^{18}$, K Venkateswara ${ }^{34}$, G Venugopalan ${ }^{6}$, A D Viets ${ }^{36}$, T Vo ${ }^{15}$, C Vorvick $^{3}$, M Wade ${ }^{37}$, R L Ward ${ }^{27}$, B Weaver ${ }^{3}$, R Weiss $^{7}$, C Whittle ${ }^{7}$, B Willke ${ }^{9,17}$, C C Wipf ${ }^{6}$, L Xiao $^{6}$, H Yamamoto $^{6}$,

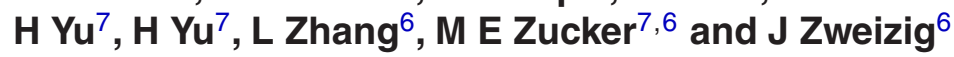

1 LIGO Livingston Observatory, Livingston, LA 70754, United States of America

2 The University of Mississippi, University, MS 38677, United States of America

3 LIGO Hanford Observatory, Richland, WA 99352, United States of America

4 Stanford University, Stanford, CA 94305, United States of America

5 Cardiff University, Cardiff CF24 3AA, United Kingdom

6 LIGO, California Institute of Technology, Pasadena, CA 91125, United States of America

7 LIGO, Massachusetts Institute of Technology, Cambridge, MA 02139, United States of America

8 University of Minnesota, Minneapolis, MN 55455, United States of America

9 Max Planck Institute for Gravitational Physics (Albert Einstein Institute), D-30167 Hannover, Germany

10 California State University Fullerton, Fullerton, CA 92831, United States of America

11 Columbia University, New York, NY 10027, United States of America

12 Louisiana State University, Baton Rouge, LA 70803, United States of America

13 Christopher Newport University, Newport News, VA 23606, United States of America

14 University of Oregon, Eugene, OR 97403, United States of America

15 Syracuse University, Syracuse, NY 13244, United States of America

16 Missouri University of Science and Technology, Rolla, MO 65409, United States of America

17 Leibniz Universität Hannover, D-30167 Hannover, Germany

18 OzGrav, University of Adelaide, Adelaide, South Australia 5005, Australia

19 RESCEU, University of Tokyo, Tokyo, 113-0033, Japan

20 OzGrav, University of Western Australia, Crawley, Western Australia 6009, Australia

21 University of Birmingham, Birmingham B15 2TT, United Kingdom

22 Universitat de les Illes Balears, IAC3 - IEEC, E-07122 Palma de Mallorca, Spain

23 SUPA, University of Glasgow, Glasgow G12 8QQ, United Kingdom

24 University of Florida, Gainesville, FL 32611, United States of America

25 The Pennsylvania State University, University Park, PA 16802, United States of America

26 University of Michigan, Ann Arbor, MI 48109, United States of America

27 OzGrav, Australian National University, Canberra, Australian Capital Territory

0200, Australia

28 Inter-University Centre for Astronomy and Astrophysics, Pune 411007, India 
29 University of Portsmouth, Portsmouth, PO1 3FX, United Kingdom

30 The University of Sheffield, Sheffield S10 2TN, United Kingdom

31 Southern University and A \& M College, Baton Rouge, LA 70813, United States of America

32 OzGrav, School of Physics \& Astronomy, Monash University, Clayton 3800, Victoria, Australia

33 The University of Texas Rio Grande Valley, Brownsville, TX 78520, United States of America

${ }^{34}$ University of Washington, Seattle, WA 98195, United States of America

35 Universität Hamburg, D-22761 Hamburg, Germany

36 Concordia University Wisconsin, 2800 N Lake Shore Dr, Mequon, WI 53097, United States of America

37 Kenyon College, Gambier, OH 43022, United States of America

E-mail: ehscwart@olemiss.edu

Received 30 July 2020, revised 11 September 2020

Accepted for publication 29 September 2020

Published 5 November 2020

\section{Abstract}

Teleseismic, or distant, earthquakes regularly disrupt the operation of ground-based gravitational wave detectors such as Advanced LIGO. Here, we present $E Q$ mode, a new global control scheme, consisting of an automated sequence of optimized control filters that reduces and coordinates the motion of the seismic isolation platforms during earthquakes. This, in turn, suppresses the differential motion of the interferometer arms with respect to one another, resulting in a reduction of DARM signal at frequencies below $100 \mathrm{mHz}$. Our method greatly improved the interferometers' capability to remain operational during earthquakes, with ground velocities up to $3.9 \mu \mathrm{m} \mathrm{s}^{-1} \mathrm{rms}$ in the beam direction, setting a new record for both detectors. This sets a milestone in seismic controls of the Advanced LIGO detectors' ability to manage high ground motion induced by earthquakes, opening a path for further robust operation in other extreme environmental conditions.

Keywords: LIGO, earthquakes, control, seismic

(Some figures may appear in colour only in the online journal)

\section{Introduction}

The detection of gravitational waves (GWs) by the Advanced Laser Interferometer Gravitational Wave Observatory (LIGO), include dozens of detections in recent years from binary black hole and neutron star mergers [1-5], which were facilitated by the use of unparalleled seismic isolation systems. At their core, the GW detectors are modified km-scale Michelson interferometers with Fabry-Perot cavities in each arm, whose mirrors must approximate inertial test masses in order to act as markers of space-time coordinates. A passing gravitational wave induces a strain in space-time, which modulates the separation of the mirrors in an arm, typically by less than $10^{-19} \mathrm{~m}$ around $100 \mathrm{~Hz}$. To measure such small displacements, the optical cavities are held on resonance [6] with highly-stabilized laser light in order to both build 
up laser power and to ensure a linear response to the GWs. Typical ground motion of $10^{-6} \mathrm{~m}$ rms requires state of the art seismic isolation to reach the small enough relative mirror motion needed to detect GWs.

The high level of isolation of the mirrors from the ground, 10 orders of magnitude at $30 \mathrm{~Hz}$ and an order of magnitude at $150 \mathrm{mHz}$, is achieved through the use of a series of passive and active control techniques [7]. The mirrors are suspended as pendula, which, in turn, are mounted on actively isolated platforms that rely on both feedforward and feedback control from sensors on the ground and the platform, respectively. Despite such phenomenal levels of isolation, particularly large seismic vibrations nonetheless remain responsible for about $5 \%$ of the unplanned downtime (out of 25\%-30\%) at both the LIGO Hanford and LIGO Livingston Observatories (LHO and LLO) during their first observing runs, O1 and O2, from 2015 to 2017. Produced by ocean waves, wind, human activity, and, most dramatically, earthquakes, seismic vibrations can cause a loss of resonance of the Fabry-Perot arms, known as a lock loss, when the input motion to the seismic control system exceeds its design capabilities. Excessive actuation to the isolation platforms exacerbates problems arising from imperfect decoupling of degrees of freedom, both on the platform itself as well as further downstream in the optical control of the cavities. After a lock loss, it takes a minimum of 30 minutes to reacquire the locked state of the detector, thus severely hindering its duty cycle.

Two fundamental types of seismic waves are generated by an earthquake: body and surface waves. Body waves propagate within a body of rock and are subdivided into the faster primary wave (P-wave), or longitudinal-compressional wave, and the slower secondary wave (S-wave), or shear wave.

When shallow earthquakes occur (at depths of several km below the surface), coupled $\mathrm{P}-\mathrm{S}$ waves known as Rayleigh waves are generated and particularly affect GW detectors because their motion is restricted to near the surface of the ground. Rayleigh waves $[8,9]$ have periods of anywhere from 3-60 s with the majority occurring at 15-20 s, corresponding to frequencies of about $50-60 \mathrm{mHz}$. The surface waves from a large earthquake can be measured around the world. These teleseismic waves impact Advanced LIGO operations when they are larger than about $1 \mu \mathrm{m} \mathrm{s}^{-1}$ at the observatories, which happens several times per week on average.

This paper focuses on the design and implementation of a novel set of techniques to reduce the detrimental effect of earthquakes on the GW detectors. Section 2 describes the active seismic isolation system used for each core mirror and highlights the problem of how the primary ground motion frequency that is amplified by earthquakes, $50 \mathrm{mHz}$, also gets amplified by the seismic control system when in its nominal configuration. Section 3 presents the solution which consists of two independent, yet complementary, modifications to the seismic control system to reduce the input signal at $50 \mathrm{mHz}$ and its amplification. Section 4 presents the results of implementing the new EQ mode at LLO and LHO during observing run 3 (O3). Finally, section 5 discusses the major accomplishments of this work and provides an outlook for the future.

\section{Advanced LIGO seismic controls}

The Advanced LIGO mirrors are suspended from isolation platforms. These platforms fulfil several roles. They provide active positioning from DC to about $30 \mathrm{~Hz}$, active isolation from ground motion from around $100 \mathrm{mHz}$ to $30 \mathrm{~Hz}$, and passive isolation above a few $\mathrm{Hz}$. The multi-stage mirror suspensions provide additional passive isolation, active damping, and control for the optics. 


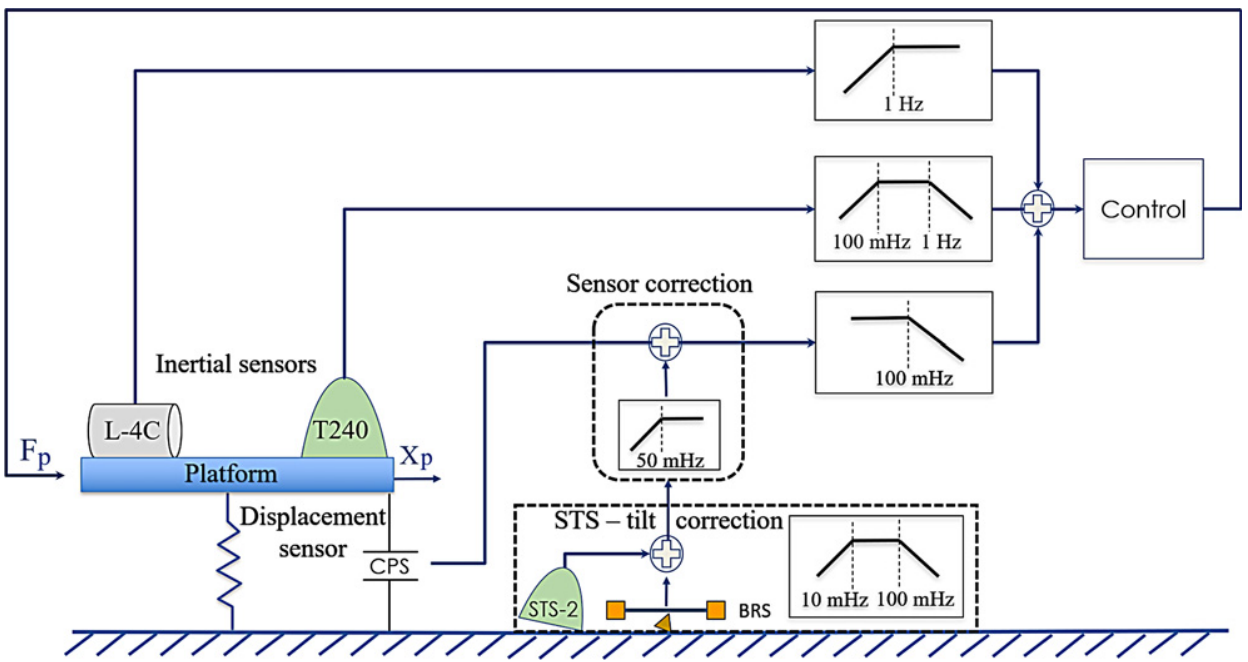

Figure 1. Illustration of the BSC-ISI stage 1 seismic controls. Sensors on the ground and on the ST1 platform are combined to act as a witness of the platform's inertial motion. Control signals are derived and sent to actuators on the platform. Note that multiple sensors (not drawn) of each type are used to provide control in all three Cartesian degrees of freedom.

The actively isolated platforms make up part of the internal seismic isolation (ISI) system, in which a number of sensors on the ground and on the platform are used for feedforward and feedback control. Each platform's motion is reduced to $10^{-12} \mathrm{~m} / \sqrt{\mathrm{Hz}}$ at $10 \mathrm{~Hz}$, several orders of magnitude below ground motion. Details about the design, operation and performance of the ISI platforms can be found in [10,11, 13].

Two types of ISI systems are used at the observatories. The HAM-ISIs are single stage platforms which support the power and signal recycling mirrors, as well as auxiliary optics, and are located in small vacuum chambers called horizontal access modules (HAMs). The BSC-ISIs are two stage platforms for the beam-splitter and the four mirrors of the arm cavities and are located in large vacuum tanks called basic symmetric chambers (BSCs) [10]. In this paper we will focus on the first stage of the BSC-ISI. It is from this stage 1 (ST1) platform that a second platform is suspended, from which the mirrors in turn are suspended ${ }^{38}$.

A schematic of the active seismic control for ST1 of the BSC-ISI is depicted in figure 1. An absolute inertial measurement of the platform motion, $X_{\mathrm{P}}$, is constructed across a broad band of frequencies from a combination of sensors on the ground and on the platform. The sensed motion is used to generate a feedback control signal, $F_{\mathrm{P}}$, which is applied to the platform via actuators to reduce the overall motion.

Information about the platform's motion above $100 \mathrm{mHz}$ is provided by two types of inertial sensors located on the platform: the Trillium 240 (T240) \& the Sercel L-4C geophone [14-16]. Information below $100 \mathrm{mHz}$ is provided by displacement sensors on the platform, the microsense capacitive position sensors (CPS), and a tilt-corrected Streckeisen STS-2 seismometer located on the floor near the BSC $[14,17]$.

\footnotetext{
${ }^{38}$ The full list of LIGO acronyms is given in [13].
} 


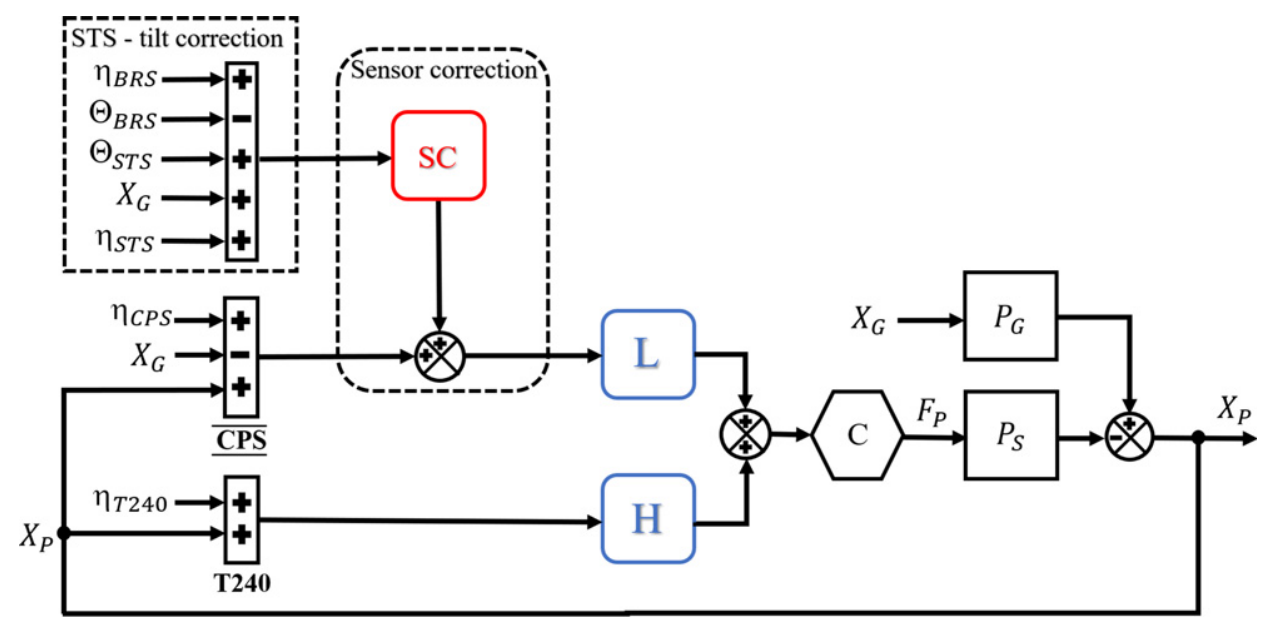

Figure 2. Block diagram of the control servo up to $500 \mathrm{mHz}$ for one degree of freedom of a BSC-ISI ST1 platform. The L-4C is not depicted because it only contributes to the control above $\sim 1 \mathrm{~Hz}$.

The CPS measures the relative position between the ground and the ST1 platform. In order to use the CPS as an inertial sensor of the platform's motion, information about the ground's absolute motion is needed. A scheme denoted as sensor correction (SC), in which the signal from the STS-2 is first high-pass filtered and then added to the CPS signal turns the CPS into a virtual inertial sensor at frequencies above $50 \mathrm{mHz}$ [18]. The filtering of the STS-2 signal, using a filter called the sensor correction filter, is necessary because at frequencies below $\sim 50 \mathrm{mHz}$, the STS-2 measurements of ground motion are contaminated both by sensor noise and by unresolved tilt of the ground due to the fact that conventional seismometers cannot differentiate between horizontal displacement and ground tilt [19]. Ground tilt that can be measured is removed from the STS-2 signal through a scheme called STS-tilt correction, which uses a beam rotation sensor (BRS) that measures the tilt of the ground near the STS-2 [19].

A block diagram detailing the design of the nominal control scheme for a BSC-ISI ST1 platform up to $500 \mathrm{mHz}$ is shown in figure 2. Inputs representing sensor noises $\left(\eta_{\mathrm{BRS}}\right.$, $\eta_{\mathrm{STS}}, \eta_{T 240}$ and $\left.\eta_{\mathrm{CPS}}\right)$ and actual ground $\left(X_{\mathrm{G}}\right)$ and platform $\left(X_{\mathrm{P}}\right)$ motion are shown on the left and the resulting inertial motion of the platform on the right. The tilt-corrected ground motion is given by $\widetilde{X}_{\mathrm{G}}=X_{\mathrm{G}}+\Theta_{\mathrm{STS}}-\Theta_{\mathrm{BRS}}+\eta_{\mathrm{STS}}+\eta_{\mathrm{BRS}}$, where $\Theta_{\mathrm{STS}}$ and $\Theta_{\mathrm{BRS}}$ is the ground tilt sensed by the STS-2 and the BRS correspondingly. These two tilt contributions cancel each other out, therefore $\widetilde{X}_{\mathrm{G}}$ becomes limited by the noise from one of the two instruments, $\eta_{\mathrm{STS}}$ or $\eta_{\mathrm{BRS}}$. The CPS signal by definition is $X_{\mathrm{P}}-X_{\mathrm{G}}+\eta_{\mathrm{CPS}}$. The sensorcorrected CPS signal is low-pass filtered (L) and blended with the high-pass filtered $(\mathrm{H})$ inertial sensors. $P_{\mathrm{S}}$ and $P_{\mathrm{G}}$ are the plant transfer functions, describing how the platform reacts to the actuation and ground motion, respectively. The control filter, $C$, is designed such that the open loop gain is unity at $\sim 30 \mathrm{~Hz}$, and is very large at low frequencies [20].

When feedback is engaged, the expected inertial motion of the platform below $\sim 1 \mathrm{~Hz}$ is thus given by 


$$
\begin{aligned}
X_{\mathrm{P}}= & \frac{P_{\mathrm{G}} X_{\mathrm{G}}+\left(X_{\mathrm{G}}-\widetilde{X}_{\mathrm{G}} \cdot \mathrm{SC}\right) \cdot L \cdot C \cdot P_{S}}{1+C \cdot P_{\mathrm{S}}}-\frac{\eta_{\mathrm{BRS}} \cdot \mathrm{SC} \cdot L \cdot C \cdot P_{\mathrm{S}}}{1+C \cdot P_{\mathrm{S}}} \\
& -\frac{\left(\eta_{\mathrm{CPS}} \cdot L+\eta_{T 240} \cdot H\right) \cdot C \cdot P_{\mathrm{S}}}{1+C \cdot P_{\mathrm{S}}}
\end{aligned}
$$

which can be simplified to

$$
\underset{C \cdot P_{\mathrm{S}} \gg 1}{X_{\mathrm{P}}}=X_{\mathrm{G}} \cdot(1-\mathrm{SC}) \cdot L-\eta_{\mathrm{BRS}} \cdot \mathrm{SC} \cdot L-\left(\eta_{\mathrm{CPS}} \cdot L+\eta_{T 240} \cdot H\right)
$$

given the very large gain $\left(C \cdot P_{\mathrm{S}} \gg 1\right)$ at frequencies below $1 \mathrm{~Hz}$ and that $L+H=1$ by construction. We further assume that $\widetilde{X}_{\mathrm{G}} \approx X_{\mathrm{G}}+\eta_{\mathrm{BRS}}$ because tilt cancels out (i.e. $\Theta_{\mathrm{STS}}$ $\left.=\Theta_{\mathrm{BRS}}\right)$ and $\eta_{\mathrm{STS}}$ is negligible with respect to $\eta_{\mathrm{BRS}}$ at frequencies below $1 \mathrm{~Hz}$.

This nominal configuration of the seismic controls is regularly used and enables operation during the majority of the varying environmental conditions, including different microseismic states, wind and small earthquakes $\left(<1 \mu \mathrm{m} \mathrm{s}^{-1}\right)$. To operate through larger earthquakes, this configuration was altered in order to confront the impact of high ground motion on the stability of the detectors.

\section{Earthquake mode}

The goal of a seismic control configuration specifically designed for use during earthquakes is to maintain the optimized performance of the seismic isolation system at frequencies above $100 \mathrm{mHz}$ while limiting gain peaking in the $50-60 \mathrm{mHz}$ frequency band [7]. The result should be a reduction of the fluctuations of the length of each arm, as well as a reduction of the differential motion of the arms with respect to one another. The latter is the degree of freedom known as DARM (differential arm) which dictates the sensitivity of the detector to GWs. $E Q$ mode consists of two distinct modifications to the ISI controls: (1) a change to the SC filter; and (2) a change to the input of the SC filter.

\subsection{The sensor correction filter}

The SC filter is a high pass filter designed to maximize the seismic isolation at $100-300 \mathrm{mHz}$, where the ground motion is dominated by the secondary microseism caused by storms in the ocean [21], while suppressing any BRS noise or residual tilt below $100 \mathrm{mHz}$. The trade off for good isolation above $100 \mathrm{mHz}$ is amplification of the ground motion at the cut-off frequency of the high pass SC filter. We call gain peaking the maximum amplification in the frequency band where the suppression function (1-SC) is greater than unity ${ }^{39}$.

The nominal SC filter results in gain peaking at $50 \mathrm{mHz}$, as shown in figure 3(a). The reasoning for the choice of the gain peaking frequency is due to low amplitude of the ground motion in this frequency band outside of earthquakes [23, 24]. Figure 3(a) also plots the ground motion measured by the STS-2 during a quiet time and during a magnitude 5.9 earthquake in Indonesia (2019-10-14 22:23:54 UTC, depth $19.0 \mathrm{~km}$ ), clearly showing that the frequency of the gain peaking coincides with the frequency of the peak energy of the earthquake. When the nominal SC filter is used during an earthquake, the amplified excess motion in the $50-60 \mathrm{mHz}$ frequency band often results in a lock loss of the interferometer.

\footnotetext{
${ }^{39}$ The Bode integral theorem, or 'waterbed theorem' shows that, for non-trivial real time control systems, improved performance at some frequencies results in degraded performance at other frequencies [22].
} 


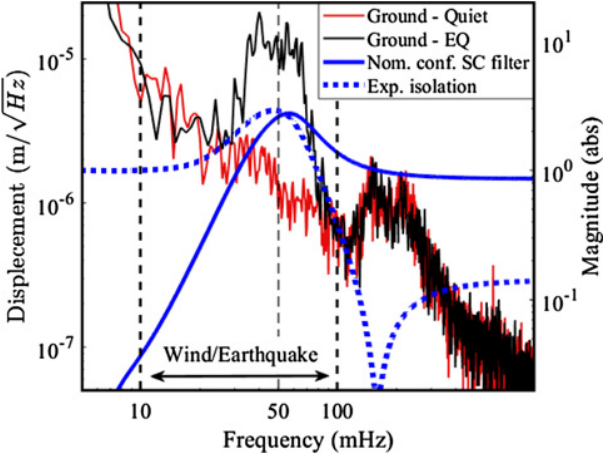

(a)

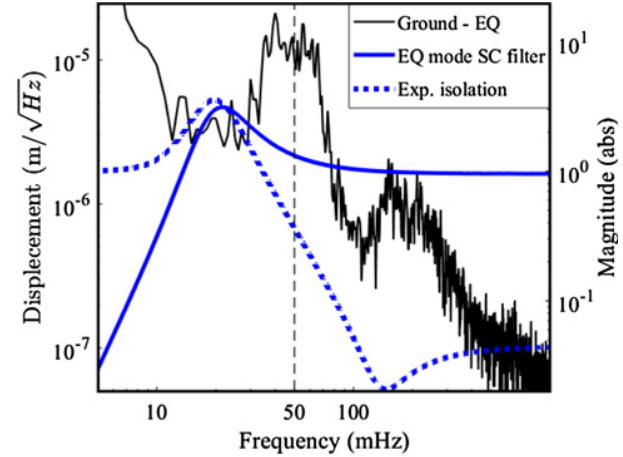

(b)

Figure 3. (a) Ground motion during an earthquake (black) and quiet times (red) as measured by an STS-2. The nominal SC filter (solid blue) has gain peaking at $50 \mathrm{mHz}$ so that $\mathrm{SC} \ll 1$ at lower frequencies to ensure that the unresolved tilt and sensor noises are decoupled from the sensor correction. The expected isolation of the ISI platform from the ground, $1-\mathrm{SC}$, is shown in dashed blue. (b) The $\mathrm{SC}$ filter for EQ mode has a gain peaking shifted out of the earthquake band down to $20 \mathrm{mHz}$. This is made possible by the decreased false signals at these frequencies during earthquakes.

A modification to the SC filter that shifts the gain peaking to lower frequencies is the first of two components that comprise the new EQ mode scheme. Figure 3(b) shows the SC filter designed for $E Q$ mode, which has a gain peak shifted down to $20 \mathrm{mHz}$. The expected isolation $(1-\mathrm{SC})$ is also plotted, highlighting how the SC filter for EQ mode provides nearly an order of magnitude more isolation at $50 \mathrm{mHz}$ compared to the nominal filter, while maintaining the requirements for frequencies above $100 \mathrm{mHz}$.

The shifted gain peaking has the effect of introducing excess motion below $30 \mathrm{mHz}$ compared to the nominal SC filter. Tilt motion of the ground and sensor noise of the BRS dominate the motion below $30 \mathrm{mHz}$ under normal conditions, therefore restricting our ability to use the modified SC filter all the time. The EQ mode SC filter can be used during an earthquake, however, because the character of the ground motion is different than during seismically quiet times. During an earthquake, translational motion of the ground, rather than tilt, dominates at frequencies around $50 \mathrm{mHz}$ [25].

\subsection{A global control scheme}

During earthquakes, using the large local ground motion as input to the sensor correction scheme can be problematic. Attempting to keep each ISI platform inertially isolated can saturate the actuators, compromising cavity stability and resulting in a lock loss. The ground motion along the length of an arm during an earthquake is largely coherent, however, and leads to the second modification that comprises $E Q$ mode: the removal of the common motion along an arm from the input to the SC filter.

An analysis of 134 earthquakes detected at LLO during O3a shows that during earthquakes the common motion of the ground from one end of an arm to the other dominates the differential motion by a factor of 5-6 in the earthquake band. Defining common-mode as $\mathrm{CM}=\left(X_{\mathrm{G}}^{I}+\right.$ $\left.X_{\mathrm{G}}^{E}\right) / 2$, and differential-mode as $\mathrm{DM}=\left(X_{\mathrm{G}}^{I}-X_{\mathrm{G}}^{E}\right) / 2$, where $I$ and $E$ represent the corner and end stations, respectively, the ground motion at each station can be represented as a linear combination of DM and CM. Because the differential motion is much smaller than the common motion during an earthquake, removing the common motion component from the input to a 


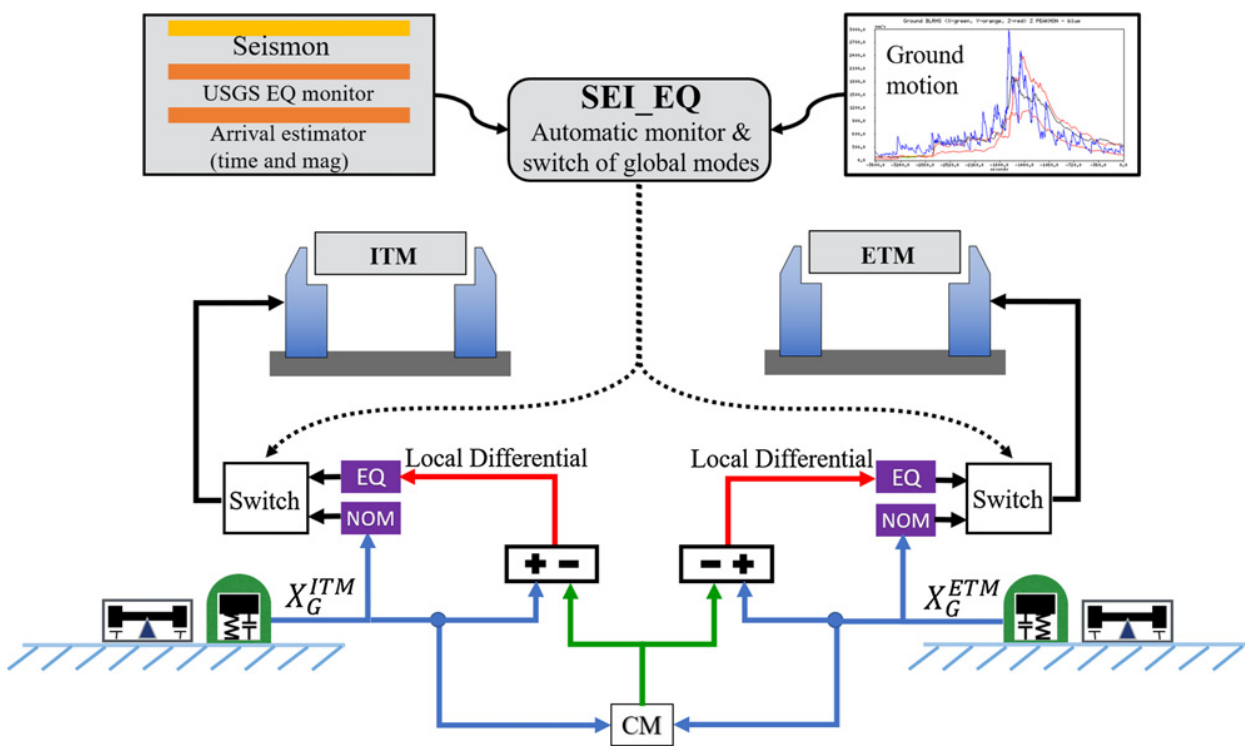

Figure 4. Global automatic seismic sensing and control of an arm cavity for one degree of freedom. The local differential is used as an input to the sensor correction of the CPS on the ST1 platform of each station during EQ mode.

platform's control system yields a control signal that falls within the maximum range of the actuators.

We therefore introduced a novel global control scheme which is illustrated in figure 4 . The STS-2 signals at the corner and end stations are used to calculate the CM motion along the arms. This is in turn subtracted from the local ground motion at each station, thus creating a local differential signal, which is used as the input to the SC filter during earthquakes. By allowing the platforms to sway together, while isolating solely against the differential motion, we create a more stable interferometer ${ }^{40}$.

Figure 5 shows the modification to the sensor correction path for $E Q$ mode. The $\mathrm{CM}$ of an arm is filtered by a low-pass filter, $\mathscr{L}_{\mathrm{CM}}$, which is designed to reject the secondary microseism $(100-300 \mathrm{mHz})$ while preserving the magnitude and phase of the common mode signal in the earthquake band, below $100 \mathrm{mHz}$. The filtered CM signal is then subtracted from the local ground motion thus giving the local differential signal, which serves as input to the sensor correction during an earthquake. Removal of the $\mathrm{CM}$ below $100 \mathrm{mHz}$ is intended to reduce the platform drive when large ground motion occurs. Without the filtering of the CM signal, the secondary microseism would add in quadrature with the local signal, as typically uncorrelated from one end to the other, and would be detrimental for the isolation performance in this band.

In figure 6(a) we present $\mathscr{L}_{\mathrm{CM}}$ along with the expected reduction of CM motion. Figure 6(b) shows the local ground motion and the resultant local differential motion after $\mathscr{L}_{\mathrm{CM}}$ is applied. Here we see one of the impacts of the new EQ mode control approach: up to $50 \mathrm{mHz}$ the input

${ }^{40}$ At VIRGO detector, a different global control scheme is used to mitigate the same issues during earthquakes depicted in this paper. The Virgo scheme is based on replacing of the differential position sensor with the differential optical measurement from the detector itself [26]. 


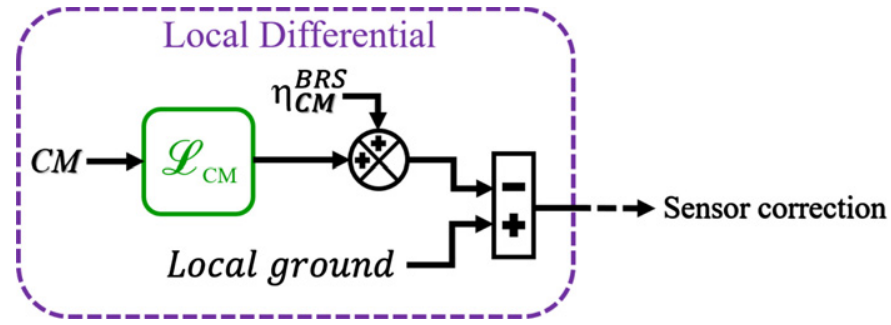

Figure 5. Block diagram depicting how the local differential signal is created. During earthquakes, the local differential signal rather than the local ground motion is used as input to the sensor correction (see figure 2). The average of the BRS noise from the two stations of the arm is given by $\eta_{\mathrm{CM}}^{\mathrm{BRS}}$.

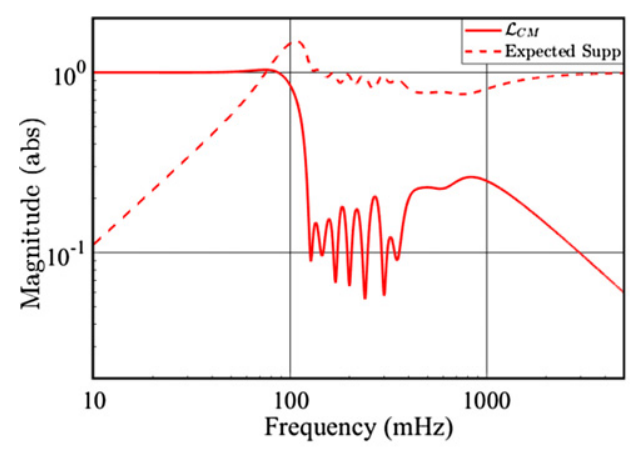

(a)

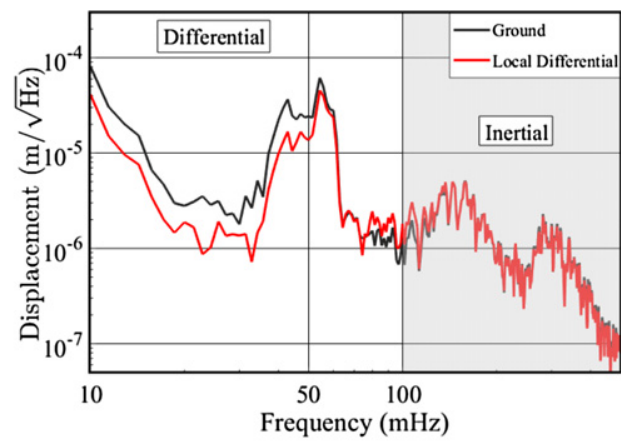

(b)

Figure 6. (a) The $\mathscr{L}_{\mathrm{CM}}$ filter (solid) with the expected CM suppression (dashed). (b) Spectra of ground motion (black) vs. local differential motion (red) created using the CM filter shown in (a).

to SC during an earthquake is reduced by a factor of 2. As seen both in figures 6(a) and (b), there is a small increase in noise near $100 \mathrm{mHz}$, however the overall reduction in input motion significantly improves the stability of the interferometer.

\subsection{The EQ mode model}

Our complete model of the ST1 platform motion for the nominal and EQ mode configurations is given by:

$$
\begin{aligned}
X_{\mathrm{P}}^{\mathrm{nom}}= & {\left[X_{\mathrm{G}} \cdot\left(1-\mathrm{SC}^{\mathrm{nom}}\right)-\eta_{\mathrm{BRS}} \cdot \mathrm{SC}^{\mathrm{nom}}+\gamma X_{\perp} \cdot \mathrm{SC}^{\mathrm{nom}}\right] \cdot L } \\
& -\left(\eta_{\mathrm{CPS}} \cdot L+\eta_{T 240} \cdot H\right) \\
X_{\mathrm{P}}^{\mathrm{EQ}}= & {\left[X_{\mathrm{G}} \cdot\left(1-\mathrm{SC}^{\mathrm{EQ}}\right)+\mathrm{CM} \cdot \mathscr{L}_{\mathrm{CM}} \cdot \mathrm{SC}^{\mathrm{EQ}}+\gamma X_{\perp} \cdot \mathrm{SC}^{\mathrm{EQ}}\right.} \\
& \left.-\left(\eta_{\mathrm{BRS}}-\eta_{\mathrm{CM}}^{\mathrm{BRS}}\right) \cdot \mathrm{SC}^{E Q}\right] \cdot L-\left(\eta_{\mathrm{CPS}} \cdot L+\eta_{T 240} \cdot H\right) .
\end{aligned}
$$

Equation (3a) describes the nominal ST1 platform motion and is identical to equation (2), except for the introduction of an additional linear term, $\gamma X_{\perp} \cdot \mathrm{SC} \cdot L$, which describes a residual horizontal motion of the platform that correlates strongly with the platform's vertical motion, 
$X_{\perp}$. The importance of the coupling term for producing a realistic model is apparent in figure 8, though the source of the coupling is not yet understood an remains and active area of investigation. The platform motion when $E Q$ mode is engaged is given by equation ( $3 \mathrm{~b}$ ). Equation ( $3 \mathrm{~b})$ includes the vertical-to-horizontal coupling term in addition to the change in the SC filter and the common-mode subtraction.

The model proves to be a powerful tool for optimizing the design of $E Q$ mode because the transfer function from ST1 to the optics can be approximated as unity below the first resonant mode frequency of the pendula $(400 \mathrm{mHz})$. We can therefore infer how the optical cavities will react in the earthquake band frequencies by simply modeling the behavior of the platforms.

To analyze the expected and actual performance of $E Q$ mode, we focus on the extent to which both the length fluctuations of a single arm cavity and DARM are reduced during an earthquake when $E Q$ mode is engaged compared to times when it is not engaged.

We can estimate the length fluctuations of an arm cavity by calculating the differential motion of the platforms of the arm along the beam direction. This is given by:

$$
\Delta_{\mathrm{P}}=\Delta_{\mathrm{G}} \cdot(1-\mathrm{SC}) \cdot L-\eta_{\Delta \mathrm{BRS}} \cdot \mathrm{SC} \cdot L+\gamma_{\Delta} \Delta_{\perp} \cdot \mathrm{SC} \cdot L+O(\text { noises })
$$

where $\Delta=\mathbf{1 - 2}$ and $O$ (noises) is the sum of all sensor noises. Importantly, $\Delta_{\mathrm{P}}$ does not include any CM contributions, yet it does rely on the SC filter. We can thus use this model to optimize the $E Q$ mode SC filter design to minimize length fluctuations of the arm during an earthquake.

Likewise, an estimate of DARM at frequencies below $100 \mathrm{mHz}$ can be made from the models for platform motion in equation (3). DARM is defined as $\left(\mathrm{ETMX}_{X}-\mathrm{ITMX}_{X}\right)-\left(\mathrm{ETMY}_{Y}\right.$ - ITMY $_{Y}$ ) Where ITM is input test mass and ETM is end test mass, thus giving:

$$
\begin{aligned}
\text { DARM }_{\mathrm{CPS}} & =\left(\mathrm{CPS}_{\mathrm{X}}^{\mathrm{ETMX}}-\mathrm{CPS}_{\mathrm{X}}^{\mathrm{ITMX}}\right)-\left(\mathrm{CPS}_{\mathrm{Y}}^{\mathrm{ETMY}}-\mathrm{CPS}_{\mathrm{Y}}^{\mathrm{ITMY}}\right) \\
& =X_{\mathrm{G}}^{\mathrm{DARM}}[(1-\mathrm{SC}) \cdot L-1]+\left[\gamma^{\mathrm{DARM}} X_{\perp}^{\mathrm{DARM}}-\eta_{\mathrm{BRS}}^{\mathrm{DARM}}\right] \cdot \mathrm{SC} \cdot L .
\end{aligned}
$$

With these sets of equations, we can predict the motion of a single optic as well as various degrees of freedom of the whole interferometer, allowing us to optimize the design of the seismic isolation system to achieve the best optical performance of the detector during earthquakes.

\subsection{Implementation and automatic switching of EQ mode}

Due to the complexity of changing the detector configuration both globally and locally in lock, a change in configuration always has the risk of contaminating the observation data or causing a lock loss. It is therefore essential to switch to EQ mode only when a problematic earthquake arrives at the site and not at other unnecessary times. For that, an early alert system named Seismon, which uses publicly available data acquired from a global network of seismic observatories compiled by USGS, was developed and installed at the observatories [27, 28]. Based on a machine learning algorithm, Seismon predicts the arrival times and velocities of the $\mathrm{P}$ and $\mathrm{S}$ body waves and, most importantly, the Rayleigh surface waves of an earthquake with a notification latency of several minutes for earthquakes more than $2000 \mathrm{~km}$ away. Using this information and the current state of the interferometer, a decision can be made about whether to engage $E Q$ mode.

Both interferometers are governed by a state machine called Guardian [29] which consists of automation nodes capable of automatic handling of control changes. We designed a Guardian sub-system (see figure 4) which monitors Seismon alerts together with the local ground motion 
in all degrees of freedom to initiate the change in configuration to $E Q$ mode when the necessary trigger levels are reached. When the earthquake has passed and the ground motion is below the nominal thresholds, the guardian transfers the seismic platform controls back to the nominal configuration. These changes of state only require a few minutes, as it is possible to switch the filters without having to turn off the seismic isolation loops, and thus keep the interferometer locked. The threshold that we have chosen to trigger EQ mode is for earthquakes that produce Rayleigh waves larger than $1 \mu \mathrm{m} \mathrm{s}^{-1}$ at LLO and $0.5 \mu \mathrm{m} \mathrm{s}^{-1}$ for LHO. Our nominal configuration is capable of handling smaller earthquakes.

When engaging $E Q$ mode, we change the seismic control configuration for not only all of the ST1 platforms in the BSC chambers, but also for the single platforms of the HAM chambers. The core optics for the power and signal recycling cavities, crucial components of the design of the GW detectors, are located in both the HAM and BSC chambers. The change to the HAMISI controls during EQ mode follows the same concepts as the changes for the ST1 platform of the BSC-ISI.

\section{Results}

A month long break in the third observing run separates $\mathrm{O} 3$ into 2 parts, named $\mathrm{O} 3 \mathrm{a}$ and $\mathrm{O} 3 \mathrm{~b}$. $E Q$ mode was first implemented at LHO at the start of O3a and at LLO at the start of O3b. We studied the performance of the isolation platforms at LLO during several earthquakes at the end of $\mathrm{O} 3 \mathrm{a}$ and beginning of $\mathrm{O} 3 \mathrm{~b}$. We verified that the model for the nominal seismic configuration fits well with the data and then used the model to optimize the design of $E Q$ mode. Here, we present the reduction we achieved in local platform motion, single arm motion and DARM when $E Q$ mode was engaged during two of the earthquakes studied. This in turn enabled the interferometer to stay locked during the high ground motion. Lastly, we give a statistical analysis of the detectors' performance before and after the implementation of $E Q$ mode.

\subsection{Local platform and single arm motion}

At LLO, one of the times EQ mode was engaged was during a magnitude 6.3 earthquake, $102 \mathrm{~km}$ WNW of Kirakira, Solomon Islands (2020-01-27 05:02:01 UTC, depth of $21 \mathrm{~km}$ ). The interferometer managed to stay locked with ground rms velocities up to $3.9 \mu \mathrm{m} \mathrm{s}^{-1}$ for the horizontal direction and $2.5 \mu \mathrm{m} \mathrm{s}^{-1}$ for the vertical, a new record for the detectors (with the nominal configuration, the maximum achieved was $2 \mu \mathrm{m} \mathrm{s}^{-1}$ for horizontal and $1 \mu \mathrm{m} \mathrm{s}^{-1}$ for the vertical direction during an earthquake). Plotted in figure 7(a) is the modeled and measured single platform horizontal motion of ITMX, $X_{\mathrm{P}}$, for the nominal configuration and EQ mode during the Solomon Islands earthquake. The EQ mode model fits well with the data, thus demonstrating it is a trustworthy tool for further optimizations. At the earthquake peak frequency of $50 \mathrm{mHz}$ we get a factor 2.6 reduction in both local platform motion and drive, when switching from the nominal configuration to EQ mode. In figure 7(b) we show the modeled contributions for $E Q$ mode displacement model, as from equation (3b). From figure 7(b), we see that at $50 \mathrm{mHz}$, the biggest contribution to the single platform motion is the commonmode motion and the vertical to horizontal coupling. Therefore the removal of common-mode was the main reason of the reduction in the single platform drive and motion.

In figure 8(a), we present the arm length fluctuations of the $\mathrm{x}$-arm, as witnessed by the T240 inertial sensors located on each platform of the arm. We also plot our models (equation (4)) for the nominal configuration and for $E Q$ mode. Furthermore, we show the model without the addition of the coupling term, to demonstrate the substantial contribution the coupling term has on the differential motion of the arm. The coupling coefficient per platform, $\gamma$, was empirically 


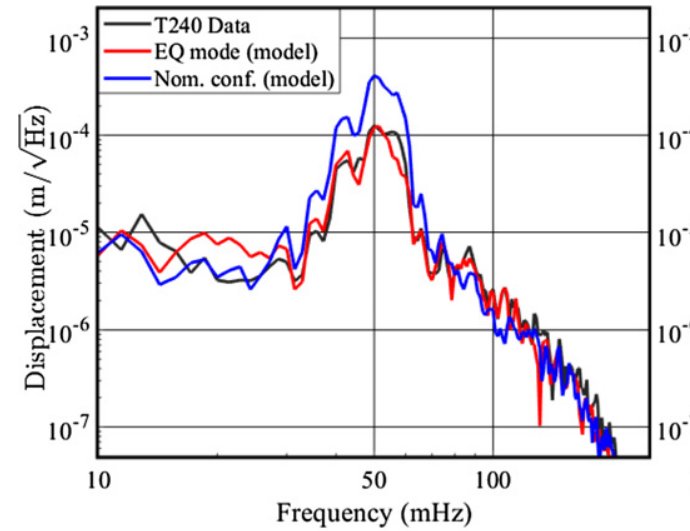

(a)

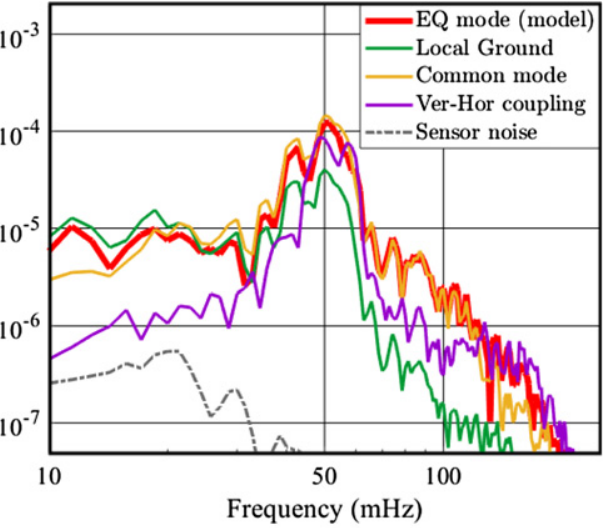

(b)

Figure 7. (a) Predicted ITMX ST1 platform motion during an earthquake for the nominal configuration (blue) and EQ mode (red). Data (black) for the platform motion (T240) was acquired when the $E Q$ mode was engaged. (b) Modeled contributions to the ST1 platform motion that are used to construct the $E Q$ mode model in (a) as from equation ( $3 \mathrm{~b}$ ). Legend in (b) contains-local ground: $X_{\mathrm{G}} \cdot\left(1-\mathrm{SC}^{\mathrm{EQ}}\right) \cdot L$, common mode: $\mathrm{CM} \cdot \mathscr{L}_{\mathrm{CM}} \cdot \mathrm{SC}{ }^{\mathrm{EQ}}$. $L$, Ver-Hor coupling: $\gamma X_{\perp} \cdot \mathrm{SC}^{\mathrm{EQ}} \cdot L$ and dashed line is the sum of all sensor noise contributions.

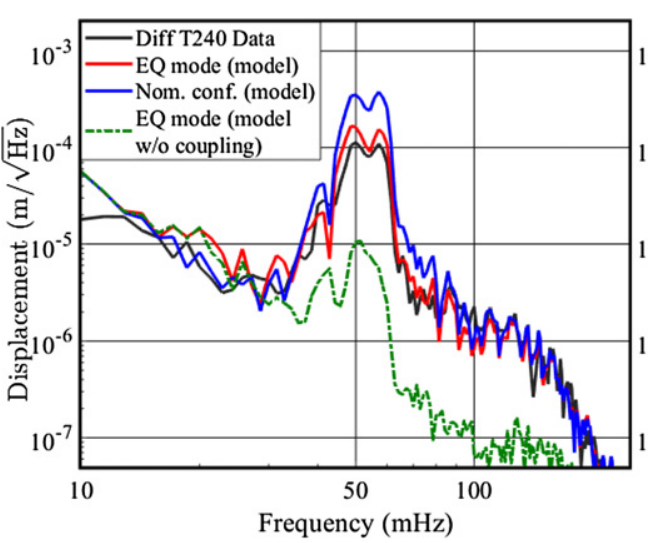

(a)

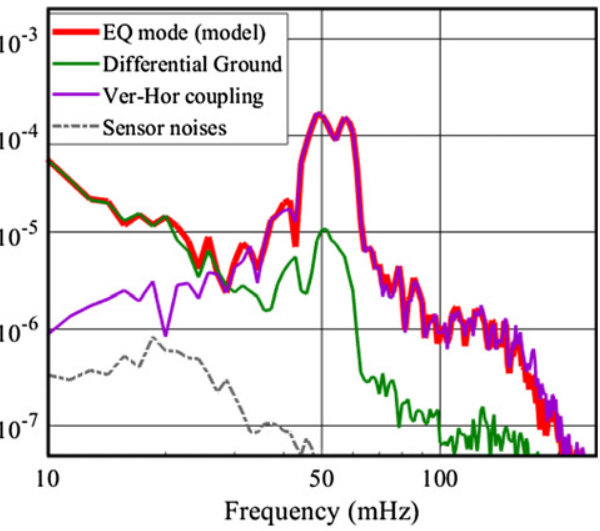

(b)

Figure 8. (a) Predicted differential ST1 platform motion along the X-arm for nominal (blue) and EQ mode (red) during an earthquake. (b) The same as in figure 7(b), detailing the arm length fluctuations model. Legend in (b) contains-local ground: $\Delta_{\mathrm{G}} \cdot(1-\mathrm{SC})$ $\cdot L$, Ver-Hor coupling: $\gamma_{\Delta} \Delta_{\perp} \cdot \mathrm{SC} \cdot L$ and dashed line is the sum of all sensor noise contributions.

determined so that the model optimally fits the data in $30-300 \mathrm{mHz}$ band. On average, $\gamma \approx$ 0.2 , but does differ between platforms at the different stations. At $50 \mathrm{mHz}$ we observe a factor 2.5 reduction of arm length fluctuations between the nominal and EQ mode configurations. In figure 8(b) as in figure 7(b), we show the modeled contributions constructing the EQ mode model in (a) as in equation (4). We see that the vertical to horizontal coupling term is the dominant contribution to the single arm differential motion. 


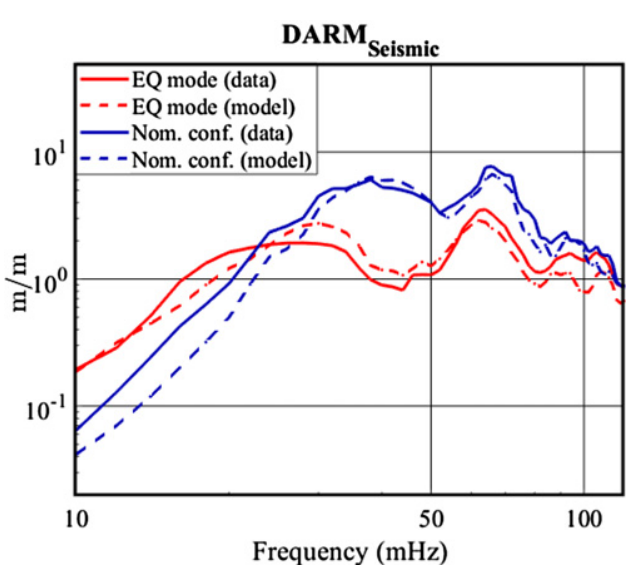

(a)

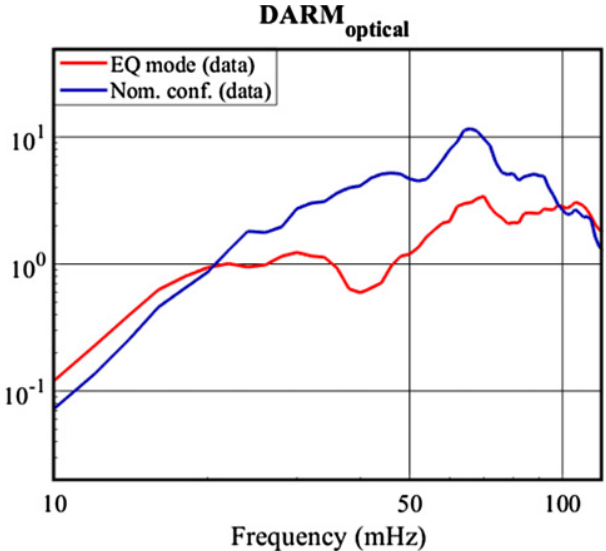

(b)

Figure 9. (a) Seismic DARM signal. Measured data in solid lines and our model (equation (5)) as dashed lines. (b) Optical DARM signal motion. In both sub figures we normalize the plots by the ground motion and blue is the nominal configuration while red is $E Q$ mode.

\section{2. $D A R M$}

To assess the performance of DARM during earthquakes, when either the nominal configuration or $E Q$ mode is engaged, we transitioned between these two configurations in the middle of an earthquake to allow close comparison for the same ground conditions and detector optical state (alignment parameters, drift in laser power and gain and optimum optical states change constantly thus affecting performance as well). The data presented is of a 6.1 magnitude earthquake in the Southern East Pacific Rise (2019-12-25 20:20:12 UTC, depth 10.0 km). The maximum rms ground velocities reached $2.3 \mu \mathrm{m} \mathrm{s}^{-1}$ for horizontal motion and $1.3 \mu \mathrm{m} \mathrm{s}^{-1}$ for vertical motion.

To track the optical signals, we observed the DARM control signal used as input to the ETMX quadruple suspension, which is used to push, or drive, the test mass in order to compensate for the differential arm motion and keep the interferometer locked. Only the top and upper intermediate (UIM) masses had significant and comparable contributions to the total control signal, while the penultimate (PUM) and bottom test mass (TST) were negligible (for LHO, it is the UIM and PUM that contribute to the DARM signal). We calibrated this DARM control signal for sub Hertz frequencies in displacement units ${ }^{41}$.

In figure 9(a), we show the seismic DARM signal together with our model (equation (5)). In figure 9(b), we plot the optical DARM signal. Both plots are normalized by the ground motion. In this example we get a factor of 4 reduction between the nominal configuration and $E Q$ mode at $50 \mathrm{mHz}$. From the figure we can see that the optical DARM signal follows the seismic DARM signal, and in turn, our models, in the earthquake band. By this, we show that we can optimize DARM performance during an earthquake by observing the seismic behavior of the platforms and use our models to tune the SC filter and the input to it, and infer from that how the optical cavity will react.

\footnotetext{
${ }^{41}$ We used a calibration based on the maximum displacement range at DC per OSEM, per our maximum digital to analog converter range of 18 bits for M0, L1 and L2 [30].
} 

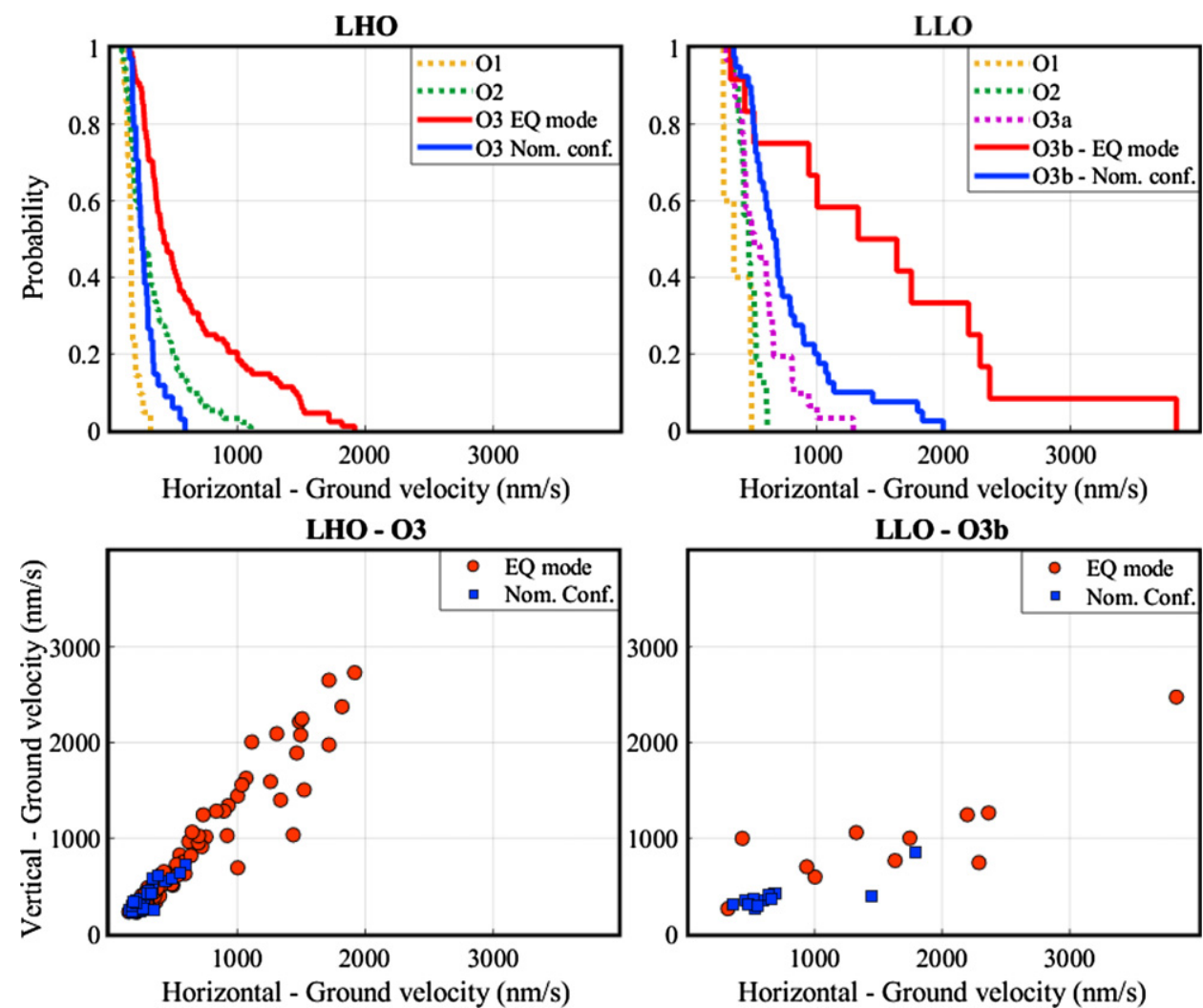

Figure 10. Top: The probabilities during each observing run for the LHO and LLO interferometers to stay locked as a function of ground velocity. EQ mode was first implemented at LHO in O3a and at LLO in O3b. Bottom: Distribution of ground velocities for the two control modes engaged during earthquakes for which the detectors remained locked during O3 (LHO-left) \& O3b (LLO-right).

\subsection{Statistical analysis}

We studied the probability of the LLO and LHO detectors to maintain lock during earthquakes for each observation run starting from O1. The results are presented in figure 10. We looked at the maximum sustained ground velocities in the beam direction during earthquakes. We included all earthquakes that had yielded a ground velocity rms greater than $200 \mathrm{~nm} \mathrm{~s}^{-1}$ in the $30-100 \mathrm{mHz}$ band, as sensed by the vertical STS-2 at all stations. We then calculated the cumulative distribution functions for each observing run. The two top figures show the clear difference between engaging $E Q$ mode versus staying in the nominal configuration during an earthquake. The probability for the interferometer to stay locked during ground velocities above $1000 \mathrm{~nm} \mathrm{~s}^{-1}$ for LLO and $500 \mathrm{~nm} \mathrm{~s}^{-1}$ for LHO has greatly increased. For smaller earthquakes, we attribute the improvement to other configuration upgrades carried out. The two bottom figures show the ground velocity distributions for both the horizontal and vertical directions for $E Q$ mode and the nominal configuration during O3 for LHO and O3b for LLO (as in the the top figures). The noticeable difference in performance of the two detectors, when using $E Q$ mode is likely due to a variety of factors. This includes but not limited to: differences 
Table 1. Summary of LLO \& LHO performance during earthquakes for pre and post integration of $E Q$ mode. White rows are data for pre-EQ mode (LLO-O3a, LHO-O2) while gray rows are for post implementation of $E Q$ mode. The threshold for defining an earthquake is $200 \mathrm{~nm} \mathrm{~s}^{-1}$ on the $30-100 \mathrm{mHz}$ band limited rms signal of vertical STS-2 at all stations.

\begin{tabular}{lccccc}
\hline Detector & Observation run & Total earthquakes & Remain lock & Lock loss & Lock probability (\%) \\
\hline \multirow{2}{*}{ LLO } & O3a & 81 & 31 & 50 & 38 \\
& O3b & 73 & 52 & 21 & 71 \\
\hline \multirow{2}{*}{ LHO } & O2 & 115 & 67 & 38 & 64 \\
& O3 & 187 & 124 & 63 & 72 \\
\hline
\end{tabular}

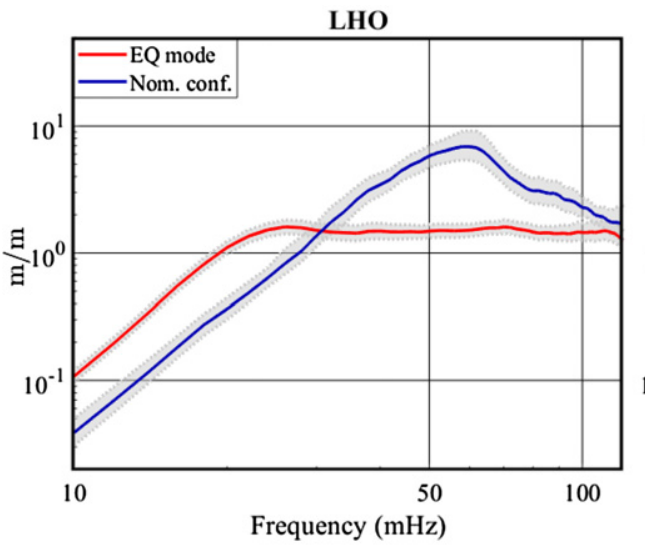

(a)

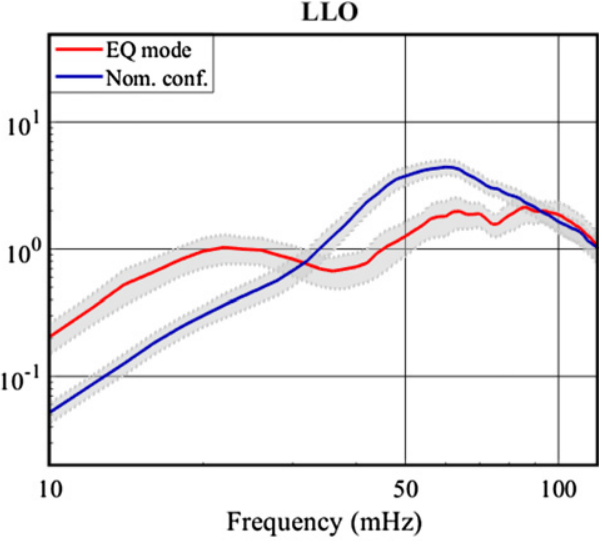

(b)

Figure 11. Averaged DARM signal normalized by ground motion during earthquakes for which the detector remained locked as given in table 1 for (a) LHO-O3 and (b) LLOO3b. The nominal configuration presented in blue and EQ mode in red. The Gray area is the $95 \%$ confidence interval of the mean for each configuration.

in the local terrain which result in more vertical ground motion at LHO compared to LLO; different control loop designs; and different point absorbers on the mirrors and different scattering sources that force different choices in alignment, which in turn affects stability [31, 32]. It should be noted that during $\mathrm{O} 3 \mathrm{~b}$, the high microseism $(100 \mathrm{mHz}-1 \mathrm{~Hz})$ posed a significant limitation to the performance at both sites, which also limited the use and effectiveness of $E Q$ mode. We do not currently have parameters in place to engage $E Q$ mode for different levels of microseism and it remains as a future goal.

In table 1 we summarize the LLO and LHO detectors' performance before (in white) and after (in gray) incorporating $E Q$ mode. For LLO, the probability of the detector to stay locked during earthquakes increased significantly from $38 \%$ in $\mathrm{O} 3$ a to $71 \%$ during $\mathrm{O} 3 \mathrm{~b}$ when $E Q$ mode was introduced. Equivalently for LHO, the increase in probability was from $64 \%$ during $\mathrm{O} 2$ to $72 \%$ during $\mathrm{O} 3$.

Lastly, in figure 11 we present a statistical analysis of the LHO and LLO DARM signals (similar to figure 9(b)) for O3 and O3b correspondingly. We averaged the calibrated DARM signal, normalized by the ground motion, over all the earthquakes for which the interferometers remained locked, as presented in table 1, separated to each seismic configuration (EQ mode is 
in red while the nominal configuration is in blue). The shaded gray area per configuration is the $95 \%$ confidence interval of the mean. This allows us to see that at $50 \mathrm{mHz}$, we get a reduction factor between the nominal configuration and EQ mode of $3.4 \pm 0.5$ for LLO and $4.2 \pm 0.6$ for LHO.

\section{Conclusions}

Earthquakes are one of the major disturbances detrimental to the performance of ground-based GW observatories, limiting their duty cycle and the amount of useful data for GW detection. In this paper we describe the design and implementation of $E Q$ mode, a modification to the nominal seismic control scheme, which includes a global control scheme to isolate against the differential motion of the detector arms, along with a change to local sensor correction filters used to decouple the isolation platforms from ground motion. We combined $E Q$ mode with a new automated system that receives alerts about incoming earthquakes and switches the detector seismic configuration to handle such events. We improved the ability of the detectors to maintain lock during ground velocities up to $3.9 \mu \mathrm{m} \mathrm{s}^{-1} \mathrm{rms}$, which is a new record.

Our seismic models predict the behavior of the local platform, the single arm differential motion and most importantly the DARM optical signal, which allows us to optimize the performance of the detector at frequencies below $1 \mathrm{~Hz}$ during an earthquake.

Analysis of the averaged optical DARM signal during earthquakes shows a clear reduction in DARM motion at frequencies below $100 \mathrm{mHz}$ upon switching from the nominal configuration to $E Q$ mode. Furthermore, our model incorporates a newly discovered cross-coupling between the platform vertical and horizontal motions, yielding a realistic model that matches well to the experimental data.

DARM performance at very low frequencies is of great importance since it directly affects the interferometer stability. Moreover, due to different processes such as light scattering from the optics, undesirable sub-hertz signals get up-converted to higher frequencies $(30-100 \mathrm{~Hz})$, and contaminate the data where the detector is most sensitive to gravitational waves. Recent investigation of the detector glitch rates during earthquakes showed no significant change between $E Q$ mode and the nominal configuration, thus allowing a continuous observing mode while switching between these two states [33].

EQ mode sets a milestone in seismic controls of the Advanced LIGO detectors, significantly reducing DARM motion at sub-Hertz frequencies during earthquakes and maintaining data quality and stability of the interferometers in extreme environmental conditions.

\section{Acknowledgments}

The authors thank the LIGO Scientific Collaboration for access to the data and gratefully acknowledge the support of the United States National Science Foundation (NSF) for the construction and operation of the LIGO Laboratory and Advanced LIGO as well as the Science and Technology Facilities Council (STFC) of the United Kingdom, and the Max Planck Society (MPS) for support of the construction of Advanced LIGO. Additional support for Advanced LIGO was provided by the Australian Research Council. This project was supported by NSF Grants: PHY-1708006 and PHY-1608922. ES acknowledge the LSC FELLOWS program for supporting his research at LIGO Livingston Observatory. LIGO was constructed by the California Institute of Technology and Massachusetts Institute of Technology with funding from 
the National Science Foundation and operates under cooperative agreement PHY-1764464 . This paper carries LIGO Document Number LIGO-P2000072.

\section{ORCID iDs}

E Schwartz (D) https://orcid.org/0000-0001-8922-7794

A Pele (D) https://orcid.org/0000-0002-1873-3769

B Lantz (D) https://orcid.org/0000-0002-7404-4845

K L Dooley (iD https://orcid.org/0000-0002-1636-0233

S Biscans (D) https://orcid.org/0000-0002-9635-7527

N Mukund (iD https://orcid.org/0000-0002-8666-9156

\section{References}

[1] Abbott B P et al (LIGO Scientific and Virgo Collaborations) 2016 Observation of gravitational waves from a binary black hole merger Phys. Rev. Lett. 116061102

[2] Abbott B P et al (LIGO Scientific and Virgo Collaborations) 2019 GWTC-1: A gravitational-wave transient catalog of compact binary mergers observed by LIGO and Virgo during the first and second observing runs Phys. Rev. X 9031040

[3] Abbott B P et al (LIGO Scientific and Virgo Collaborations) 2017 GW170817: Observation of gravitational waves from a binary neutron star inspiral Phys. Rev. Lett. 119161101

[4] Abbott B P et al (LIGO Scientific and Virgo Collaborations) 2020 GW190521: A binary black hole merger with a total mass of $150 M_{\odot}$ Phys. Rev. Lett. 125101102

[5] Abbott B P et al (LIGO Scientific and Virgo Collaborations) 2020 GW190814: Gravitational waves from the coalescence of a 23 solar mass black hole with a 2.6 solar mass compact object Astrophys. J. Lett. 894 L44

[6] Staley A et al 2014 Achieving resonance in the advanced LIGO gravitational-wave interferometer Class. Quantum Grav. 31245010

[7] Biscan S et al 2018 Control strategy to limit duty cycle impact of earthquakes on the LIGO gravitational-wave detectors Class. Quantum Grav. 35055004

[8] Bullen K E and Bolt B A 1986 An Introduction to the Theory of Seismology 4th edn Geophysical Journal of the Royal Astronomical Society 86: (Cambridge: Cambridge University Press) $215-216$

[9] Stein S and Wysession M 2003 An Introduction to Seismology, Earthquakes and Earth Structure (Oxford: Blackwell)

[10] Matichard F et al 2015 Seismic isolation of advanced LIGO: review of strategy, instrumentation and performance Class. Quantum Grav. 32185003

[11] Abbott R et al 2002 Seismic isolation for advanced LIGO Class. Quantum Grav. 191591

[12] Abbott R et al 2004 Seismic isolation enhancements for initial and advanced LIGO Class. Quantum Grav. 21 S 915

[13] Shoemaker D, Burtnyk K and Barneo P 2018 LIGO-VIRGO-KAGRA (LVK) abbreviations and acronyms list Technical Report LIGO-M080375 LIGO-Virgo collaboration

[14] Ackerley N 2014 Principles of broadband seismometry Encyclopedia of Earthquake Engineering (Berlin: Springer)

[15] Geophopnes-Seismic Sensors, Sercel Inc, 17200 Park Row Houston, Texas 77084, U.S.A. 2016 https://www.sercel.com/products/Pages/seismometers.aspx

[16] Kirchhoff R et al 2017 Huddle test measurement of a near Johnson noise limited geophone Rev. Sci. Instrum. 88115008

[17] Poduje N S and Mallory R S 1990 A.C. capacitive gauging system US Patent 4,918,376

[18] Lantz B T 2012 Description of the sensor correction FIR and IIR filter components Technical Report LIGO-T1200285

[19] Ross M P et al 2018 Low-frequency tilt seismology with a precision ground-rotation sensor Seismol. Res. Lett. 89 67-76 
[20] Matichard F et al 2015 Advanced LIGO two-stage twelve-axis vibration isolation and positioning platform. Part 2: experimental investigation and tests results Precis. Eng. 40 287-97

[21] Cessaro R et al 1994 Sources of primary and secondary microseisms Bull. Seismol. Soc. Am. 84 $142-8$

[22] Bode H W 1945 Network Analysis and Feedback Amplifier Design (New York: D. Van Nostrand Co)

[23] Peterson J 1993 Observations and modelling of seismic background noise US Geological Survey, Open-File Report 93-322

[24] Berger J, Davis P and Ekström G 2004 Ambient earth noise: a survey of the global seismographic network J. Geophys. Res. 109 B 11

[25] Schwartz E 2020 Improving IFO robustness during earthquakes Technical Report LIGO-G1902080

[26] Majorana E 2017 Ruggi's global inverted pendulum control Technical Report LIGO-G1700891

[27] Coughlin M et al 2017 Limiting the effects of earthquakes on gravitational-wave interferometers Class. Quantum Grav. 34044004

[28] Mukund N et al 2019 Ground motion prediction at gravitational wave observatories using archival seismic data Class. Quantum Grav. 36085005

[29] Rollins J G 2016 Distributed state machine supervision for long-baseline gravitational-wave detectors Rev. Sci. Instrum. 87094502

[30] Kissel J S and Roberston N A 2014 ETM/ITM quad suspension control ranges Technical Report LIGO-T1100595

[31] Buikema A et al 2020 Sensitivity and performance of the advanced ligo detectors in the third observing run (arXiv:2008.01301[astro-ph.IM])

[32] Soni S et al 2020 Reducing scattered light in LIGO's third observing run (arXiv:2007.14876[astro-ph.IM])

[33] Beda R 2020 Assessing EQ mode impacts on LLO DQ Technical Report LIGO-G2001049 